\title{
Analysis of HCV Isolates Among the Li Ethnic in Hainan Island of South China Reveals Their HCV-6 Unique Evolution and a New Subtype
}

\author{
Tao Wu ${ }^{\text {a,f }}$ Zengbo Xing ${ }^{b}$ Manqiong Yuan ${ }^{c}$ Jun Ge ${ }^{a}$ Guosheng Yuan ${ }^{f}$ \\ Kaijian Liang ${ }^{d}$ Biao $\mathrm{Wu}^{\mathrm{a}}$ Furong $\mathrm{Xiao}^{\mathrm{a}}$ Chunhua $\mathrm{Li}^{f}$ Yuanping Zhou ${ }^{\mathrm{e}}$ \\ Feng Lin ${ }^{\mathrm{a}}$ Ling Lu $\mathrm{Lu}^{\mathrm{f}}$
}

\begin{abstract}
aDepartment of Infectious Disease, Hainan General Hospital, Haikou, 'Department of Infectious Disease,Wenchang City People's Hospital of Tongji Medical College, Wenchang, 'School of Public Health, Xiamen University, Xiamen, dDepartment of Internal Medicine, Baisha People's Hospital, Baisha, e'Department of Infectious Disease, Southern Medical University, Guangzhou, China, ${ }^{\mathrm{f} C e n t e r}$ for Viral Oncology, Department of Pathology and Laboratory Medicine, University of Kansas Medical Center, Kansas, USA, gLaboratory for the Study of Hepatology, Third Affiliated Hospital of Sun Yat-sen University, Guangzhou, China
\end{abstract}

\section{Key Words}

$\mathrm{HCV} \cdot$ Full-length genome $・$ New genotype $6 \cdot$ Sequence $\cdot$ Hainan Island

\begin{abstract}
Background/Aims: Hainan Island has been inhabited by the "Li" aboriginal minority for centuries where the HCV genotype distribution patterns maybe remarkably different from other parts of China. We aimed to provide a better understanding of the infection with HCV genotype 6 among "Li" aboriginals on Hainan Island. Methods: Firstly,using RT-PCR and DNA sequencing to determined 517 partial HCV Core-E1(115 from Li Ethnic ,402 from Han Ethnic) and 8 full-length genomes from Li ethnic in Hainan Island successfully, and then using the phylogenetic tree to determine the HCV genotype distribution and analyze the evolution of them. Results: Phylogenetic tree analysis showed that the distribution pattern of HCV genotypes among the Han and Li ethnic population exhibits significant differences:6a was the most prevalent subtype in Han ethnic of Hainan Island followed by $1 b, 3 b, 2 a, 3 a$, and $1 a$. All genomes from Li ethnic were classified into genotype 6 , while 84 out of $115(73 \%)$ could not be classified. Nine sequences (HN1350 et al.) from Li ethnic might be assigned to a new subtype $6 x h$ as their $p$-distances ranged from 5.9 9.7\%. Furthermore, we sequenced and characterized full-length genomes for eight HCV-6 isolates which were all from Li ethnic in Hainan Island. Among these isolates, the HN1350 was classified as a new subtype: $6 x h$. Conclusion: Overall,
\end{abstract}

T. Wu, Z.-B. Xing and M.-Q. Yuan contributed equally to this work.

\begin{tabular}{ll}
\hline Feng Lin & Department of Infectious Disease, Hainan General Hospital \\
and Ling Lu & Haikou City, Hainan Province (China) \\
& E-Mail Lin_fenghn@126.com; Ilu@kumc.edu
\end{tabular}


Subtype

we firstly defined a new subtype of genotype $6 x h$ through partial and new full length genome. And we found a unique distribution pattern of HCV 6 in the Li tribe, which might provide a better way to understand the genetic diversity of HCV-6 and to investigate the phylogeny of HCV strains from Li tribe.

\section{Introduction}

Chronic hepatitis $\mathrm{C}$ virus (HCV) infection is a major global health problem that affects 130 170 million people worldwide and represents a major cause of liver cirrhosis and hepatocellular carcinoma (HCC) [1-3]. HCV is classified into the Hepacivirus genus of the Flaviviridae family and has a high degree of genetic diversity. Based on such diversity, the virus is now classified into seven genotypes, 97 subtypes (including 84 confirmed and 13 provisionally assigned), and $>20$ lineages that display nucleotide differences equivalent to new subtypes yet unassigned [4-7]. HCV genotypes have different geographic distribution patterns and may respond differently to antiviral therapy. Although the advent of directacting antivirals (DAAs) may show improved genotype coverage and response, their approval remain restricted to specific genotypes $[8,9]$.

The HCV genotypes have different geographic distribution patterns and respond in a different way to antiviral therapy. Generally, subtypes $1 \mathrm{a}, 1 \mathrm{~b}, 2 \mathrm{a}, 2 \mathrm{~b}$, and $3 \mathrm{a}$ are globally distributed. In contrast, most other subtypes are restricted to certain regions. Recently, a wide-ranging study of HCV infection in China revealed that genotype $1 \mathrm{~b}$ is predominant (56.8\%, 582/997), followed by genotype 2 (24.1\%, 240/997), $3(9.1 \%, 91 / 997)$, and 6 $(6.3 \%, 63 / 997)$ [10]. However, our recently studies in Guangzhou found that $6 \mathrm{a}$ is the second most predominant subtype with a $20.2 \%$ prevalence, and accounts for the major proportion $(35 \%)$ in Hainan Island, indicating that genotype 6 has displaced genotype 2a and became the second genotype in South China [11].

Hainan Island has been inhabited by the "Li" aboriginal minority for centuries and this characteristic, together with its unique geographic location (Fig. 1), making it likely that the HCV genotype distribution among the population on this island is remarkably different from that observed in mainland China. The phylogenetic analysis of a few isolates from Li ethnic in our previous study indicated that the source of the Hainan HCV 6 type may be derived from older strains, so understanding genotypes and molecular epidemiology of $\mathrm{HCV}$ Hainan Island will help to understand the $\mathrm{HCV}$ genotype 6 in the origins of Southeast Asia [11]. In the current study, we aimed to provide a better understanding of $\mathrm{HCV}$ genotype 6 infection on the Li aboriginal minority in Hainan Island, so that the

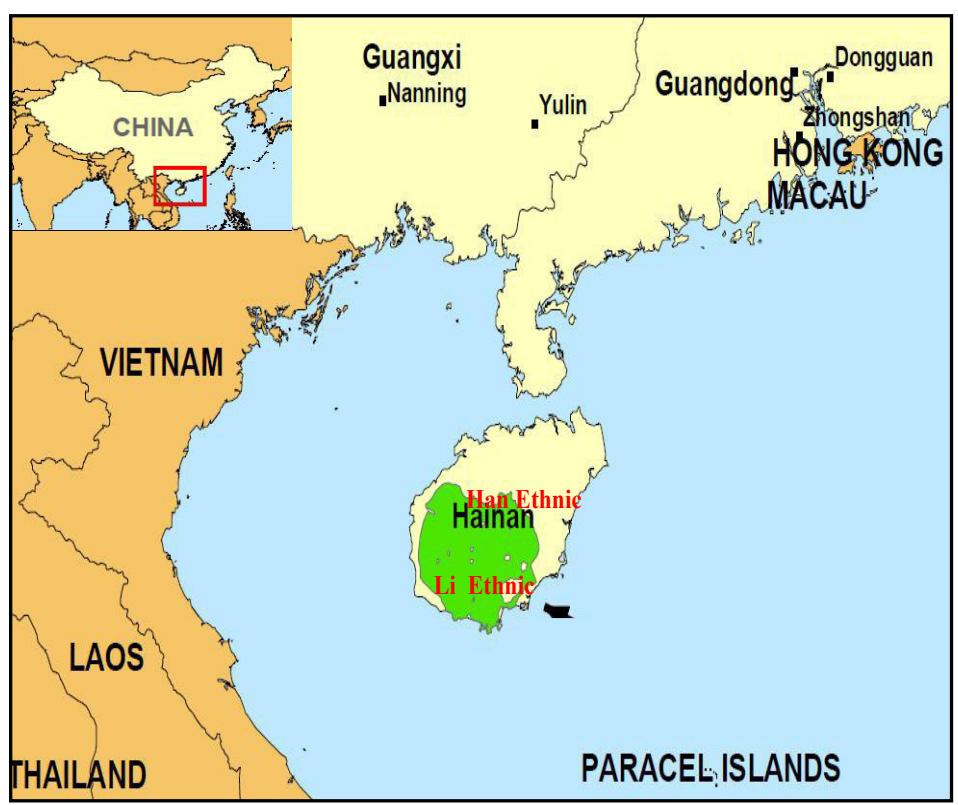

Fig. 1. The geographic location of Hainan Island in Southeast Asia.This island is located in the South China Sea in proximity to Southeast Asian countries. It has been inhabited for centuries by the "Li" aboriginal minority in the central southern mountainous region (green), while the "Han" majority occupy the northern coastal region (blank). 


\section{Cellular Physiology Cell Physiol Biochem 2018;50:1832-1839

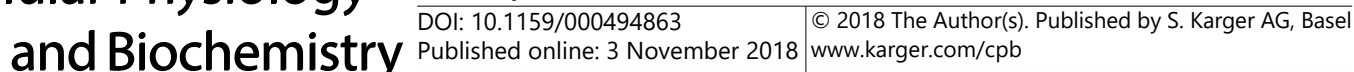 \\ Wu et al.: Unique Evolution of HCV Among the Li Ethnic in Hainan Island and a New Subtype}

information would help improve strategies for HCV prevention and control on the island and in the wider country.

\section{Materials and Methods}

\section{Subjects and samples}

517 HCV RNA positive serum samples were collected from the Hainan General Hospital and Baisha People's Hospital, between January 2013 and June 2018 (402 cases were from the Han People,115 cases were from the Li People).

\section{Study assessments}

Anti-HCV was assayed using the third-generation enzyme immunoassay reagents from Kehua Biotech Co. Ltd. HCV RNA was quantified using the Roche COBAS ${ }^{\circledR}$ TaqMan ${ }^{\circledR}$ assay (Roche Molecular Diagnostics,Pleasanton, CA, USA) with a LLOQ of $25 \mathrm{IU} / \mathrm{mL}$.The ethical review committee of the General Hospital of Hainan Province had approved this study. Guidelines set by this committee were strictly followed and written informed consent was obtained from each patients.

\section{Sequence determination}

HCV sequences were amplified in Core-E1 region (corresponding to the nucleotides numbered 7391310 in the H77 genome), following the protocols previously described [5]. Briefly, RNA was extracted in $100 \mu \mathrm{l}$ of serum using the QIA viral RNA kit (Qiagen, Valencia, CA); cDNA was converted using random hexamers and RevertAid First Strand cDNA Synthesis Kit (Fermentas Life Science, EU). An in-house RT-PCR system was used with degenerate HCV-6 primers which we have previously described [5]. Amplification was completed using conventional PCR and the amplicons were purified using the QIAquick PCR Purification Kit (Qiagen, Valencia, CA). Sequencing was done in both directions using the ABI Prism Big Dye 3.0 terminators on an ABI Prism 3500 genetic analyzer. The resulting chromatograms were visually inspected and sequences were assembled using SeqMan, from which the encoded amino acid sequences were deduced using EditSeq. Sequence alignments were made using MegAlign. These software programs are contained in the Lasergene 8.1 package (DNASTAR Company,Madison,Wisconsin).

Full-length sequence PCR amplification and sequencing were also performed to define new subtypes. Based on the resulting cDNA above, HCV genomic fragments were amplified to overlap nearly all the fulllength genome for eight isolates (HKP7, HKP16, HK25, HK26, HK38, HK43, HN1316 and HN1350). An in-house RT-PCR system was used with degenerate HCV-6 primers [12], or in combination with specific primers designed based on the determined sequences. Standard procedures were implemented to avoid potential carryover contamination [13].

\section{Phylogenetic analysis}

A full-length HCV sequence dataset was assembled by combining the eight new full-length genomes from this study with an additional 99 HCV-6 sequences and one outlier reference sequence (1a.US.1977.H77C.AF011751) (see Fig. S1 for detail - for all supplemental material see www.karger. com/10.1159/000494863/). Another dataset comprising 155 sub-genomic Core-E1 sequences was also assembled, corresponding to nucleotide positions 739-1310 in the $\mathrm{H} 77$ genome. It contained 115 sequences obtained from this study, 72 HCV-6 sequences retrieved from Genebank and two outliers (1a.US.1977.H77C. AF011751 and 2a.JFH-1). These datasets were then aligned using BioEdit followed by a manual adjustment, with the inclusion of references selected from GenBank for best representation. Prior to the phylogenetic tree reconstruction, the best-fitting substitution model was selected using the jModeltest program on the basis of the Akaike information criterion. Substitution model $\mathrm{GTR}+\mathrm{I}+\Gamma$ was found to be the most appropriate for all the resulting sequence datasets. Based on this model, the maximum likelihood (ML) trees were heuristically searched using the subtree pruning and regrafting (SPR) algorithm and the nearest-neighbor interchange (NNI) perturbation algorithm implemented in the MEGA 6.0 software, with which bootstrap analyses were performed in 1000 replicates. After tree files were generated in the Newick format, the tree topologies were displayed using the FigTree program. 


\section{Cellular Physiology Cell Physiol Biochem 2018;50:1832-1839

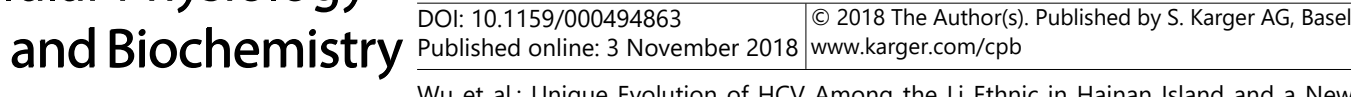 \\ Subtype}

To exclude potential recent viral recombination events, RDP3 software [14] was run for examining full length genome sequence with settings as previously described [12].

Nucleotide sequence accession numbers

The nucleotide sequences reported in this study were deposited in GenBank with the following accession numbers: MG878999-MG879000,KJ470620-KJ470625.

\section{Results}

Analysis of Core-E1 region

HCV-RNA of 517 isolates (402 cases were from the Han People,115 cases were from the Li People) were successfully extracted, amplified in the HCV Core-E1 region, and sequenced using the phylogenetic tree to analyze and determine the HCV genotype distribution of the Han and Li People in Hainan.The results of phylogenetic tree analysis showed that the distribution pattern of HCV genotypes among the Han and Li ethnic population exhibits significant differences:In the 402 isolates from Han ethnic population,31.6\% (127/402) were $1 \mathrm{~b}$, followed by genotype $1 \mathrm{~b}(33.1 \%, 125 / 402), 3 \mathrm{~b}(18.4 \%, 74 / 402), 2 \mathrm{a}(9.4 \%, 38 / 402)$, 3a $(7.5 \%, 30 / 402)$ and $1 \mathrm{a}(2 \%, 8 / 402)$. However, all genomes from Li ethnic were classified into genotype 6 .

Furthmore,with reference to those retrieved from GenBank, all the 115 sequences from $\mathrm{Li}$ ethnic were classified into 30 assigned HCV-6 subtypes, 6a-6xh (description of 6xh will be given later), and into other unclassified HCV-6 lineages. In Fig. S1, the phylogenetic tree showed that there were the majority could not be classified into known HCV genotype 6 subtypes. Among the 22 classified genomes, HN1825, HN1826, HN1614 and HN1827 were classified into 6e (p-distance from GX004=3.4 5.6\%), HN1615 into 6r (p-distance from QC245=8.4\%), HN1603, HN1604, HN1801 and HN1802 into 6w (p-distance from gz52557=14.1\% and $14.6 \%$, respectively), HN1606 and HN1607 both into 6xa (p-distance form DH012=2.7\% and $4.2 \%$, respectively), HN1835 HN1844 into $6 \mathrm{n}$ (p-distance from KM42=2 4.0\%) and HN1613 into $6 \mathrm{v}$ (p-distance from NK046=7.0\%). According to the current criteria of HCV classification, we classified the above 22 genomes into five assigned subtypes as their p-distance were within the range of $<15 \%$. Furthermore, nine new sequences in this study were closely related (HN1411, HN1717, HN1718, HN1701, HN1304, HN1350, HN1720, HN1823, and HN1824), with p-distance ranged from 5.9 9.7\%, which meets one of the current subtype classification criteria.

\section{Analysis of full-length genomes}

Full-length genome sequences were selected to characterize for eight isolates: HKP7, HKP16, HK25, HK26, HK38, HK43, HN1316 and HN1350, each with 19-22 overlapping fragments. These genomes were 9420-9439 nt in length, starting from the extreme 5'UTR end through to the variable region of the 3'UTR. Each had a single ORF of length 9039-9063 nt. The 5'UTRs were 340-342 nt long, while the 3'UTR lengths varied from 32 to $40 \mathrm{nt}$. The sizes of the other eight protein genes were consistent with those of the H77 strain with the exception of the E2 (364-371 aa) and NS5A (448-449 aa) genes.

A maximum likelihood (ML) tree was reconstructed based on eight full-length HCV genotype 6 genome sequences, together with 93 reference genomes to represent the HCV-6 subtypes an outlier group (Fig. S2). Based on phylogenetic analysis and distancebased method [14], the 93 HCV- 6 sequences were divided into 31 subtypes, 6a-6xf, and 29 unassigned lineages. Each of these exhibited full bootstrap support of $100 \%$ in the phylogeny. The two newly-generated sequences from this study, HN1316 and HN1350 would be independently separated from other isolates. These two genomes, which were all sampled in Li ethnic, showed pairwise nucleotide similarities to their nearest references of $71.42-79.13 \%$, and $70.78-74.71 \%$, respectively. Based on the criteria of the current HCV classification system [15], the genetic similarities are sufficiently high to classify these two 


\section{Cellular Physiology Cell Physiol Biochem 2018;50:1832-1839

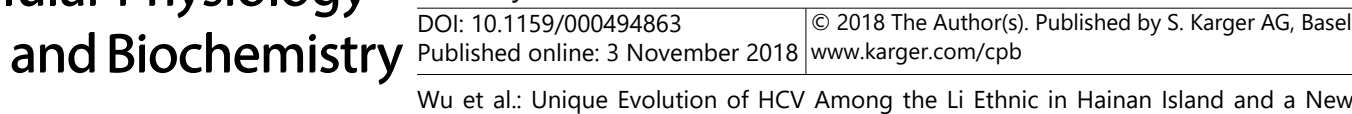 \\ Subtype}

isolates into the six subtypes outlined above. Moreover, as for HN1350, there were more than three closed related isolates. Therefore, with the completion in this study of the genomes for isolate HN1350, subtype 6xh is now classified by full-length sequences.

New subtype 6xh

In this study, nine HCV-6 variants (HN1411, HN1717, HN1718, HN1701, HN1304, HN1350, HN1720, HN1823, and HN1824) were clustered to be a new subtype which was named 6xh, as subtype 6xf was assigned in 2016 [4] and no other new HCV-6 subtype has been revealed since then. Phylogenetic analysis for Core-E1 region presented that the nucleotide distances among the nine sequences were less than 15\% (5.9 9.7\%), while they showed $>15 \%$ distance from the other HCV-6 clusters. Moreover, a full-length genome has also been obtained for HN1350.Therefore, according to the current recommended criteria for HCV classification, the assignment for the new subtype 6xh could now be confirmed.

\section{Similarity plotting}

To exclude the possibility of recent viral recombination, pairwise nucleotide similarity values were plotted along HCV genomes using the RDP3 software [14]. After comparing the 2 full-length genomes that were determined in this study with each other, and with all the reference sequences shown in Fig. S1, no such evidence was detected (data not shown).

\section{Discussion}

Hainan Island has been inhabited for centuries by the "Li" aboriginal minority in the central southern mountainous region (14.73\%), while the "Han" majority occupies the northern coastal region (83.33\%). In this study, we have successfully determined partial Core-E1 sequences of HCV from 115 individuals residing Li ethnic tribe and 402 individuals residing Han ethnic in Hainan Island. The phylogenetic tree showed that all the 115 isolates were classified into genotype 6, while 84 out of 115 (73.0\%) could not be classified into any known subtypes. In this study, two ethnic populations of people, the Han and Li in Hainan, seems to have different modes of transmission routes. In one of our previous study, geographical phylogenetic analysis showed that HCV in the Han population of Hainan Island mainly originated from the Chinese mainland [11]. It suggests that the transmission routes of the HCV in the Han population are part of the whole transmission cycle of $\mathrm{HCV}$ in the mainland of China. However, the present study found that the HCV genotype distribution of the Li nationality on this island is remarkably different from that observed in Han nationality, indicating a unique HCV origin and migration pattern of the Li minority. The HCV in the Li population clustered together and expanded independently along evolution history, which suggests that the HCV genotype 6 in the Li ethnic population lived in a closed-off type of life style. The virus had to evolve over a very long period of history.

Among the seven genotypes of HCV, genotype 6 (HCV 6) exhibits the highest genetic diversity $[16,17]$. Currently, a total of 31 subtypes, denoted $6 \mathrm{a}-6 \mathrm{xf}$, are formally assigned to HCV 6, and each has at least one full-length genome determined. In addition, 19 lineages have also been completely sequenced, and many novel variants have been detected through the sequencing of partial genomic regions [4, 5, 7, 18, 19]. Geographically, HCV 6 isolates are typically found in Southeast Asia or among expatriates from this region, which may suggest that this region represents the ancestral and endemic region of HCV 6 [18, 20-25]. According to evolutionary analysis, the HCV 6 originated at about 1100-1350 year ago in central Asia, and then spread slowly to the surrounding areas [26]. Our current study found that the presence the unclassified HCV 6 in the Li tribe on Hainan Island's is as high as $90.4 \%$, which is quite different from other Southeast Asia countries, including Laos, Vietnam, Cambodia, and Burma [27-29] . Besides, the original HCV 6 strain of the Li tribe of Hainan Island cannot be clustered with the currently known HCV 6 subtypes. Most current scholars (http://en.wikipedia.org/wiki/Li_ people) believe that Li moved from the East Indies by the ancient Malays National to Hainan Island, and evolved from PLN Virginia (Malaya) and Thais composition. In addition, previous 


\section{Cellular Physiology Cell Physiol Biochem 2018;50:1832-1839

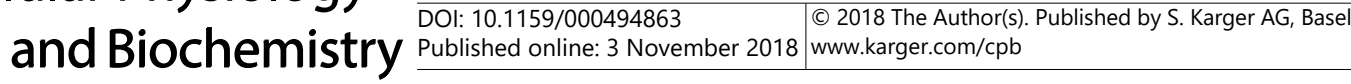 Wu et al.: Unique Evolution of HCV Among the Li Ethnic in Hainan Island and a New Subtype}

study for HCV $6 \mathrm{~g}$ and $6 \mathrm{w}$ sequence analysis in Li tribe found that the $6 \mathrm{~g}$ and $6 \mathrm{w}$ of Li tribes were substantially originated before the 6th century, indicating HCV 6 endemic spreading in $\mathrm{Li}$ has been popular and evolved for a very long time [30]. One possible speculation could be: As an ethnic minority in China, the Li population is a unique group who live only off the coast of southern China on Hainan Island for approximately 5000 years. Most of the Li people have remained relatively isolated from other populations [11], leading HCV 6 high distribution and percentage in Li tribe and evolved as completely different from other regions of HCV 6 to be "specific viral strains".

The HCV classification system was updated in 2005 and expanded recently and provides detailed guidelines for defining new subtypes $[6,15]$. Based on the criteria of the current HCV classification system, the genetic similarities of the two newly-generated sequences (HN1316 and HN1350) are sufficiently high to classify these two isolates into the six subtypes outlined above. Moreover, as HN1350 has more than three closed related isolates, subtype $6 \mathrm{xh}$ is now classified by full-length sequences.

\section{Conclusion}

In this study, our finding might provide a better way to understand the genetic diversity of HCV-6 and to investigate the phylogeny of HCV strains from Li tribe. As a step towards a comprehensive understanding of HCV-6 diversity and origin of the Li population on Hainan Island, future studies should include larger random sampled sequences and more comprehensive clinical data.

\section{Acknowledgements}

This study was supported by the grant from Hainan Provincial government (Grant No.14A21023, ZDXM2015072 and ZDYF2017130) and National Natural Science Foundation of China (No. 81860290). Ling Lu was supported by a grant from the National Institute of Allergy and Infectious Diseases (5 R01 AI080734). Manqiong Yuan was supported by a grant from National Natural Science Foundation of China (No. 81402768). The funding agencies had no role in study design, data collection and analysis, decision to publish, or preparation of the manuscript.

\section{Disclosure Statement}

The authors declare to have no competing financial interests.

\section{References}

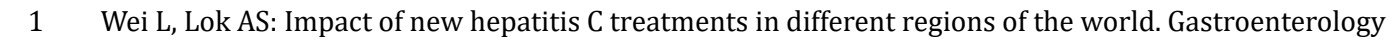
2014;146:1145-1150.

-2 Wang FS, Fan JG, Zhang Z, Gao B, Wang HY: The global burden of liver disease: The major impact of China. Hepatology 2014;60:2099-2108.

3 Cui Y, Jia J: Update on epidemiology of hepatitis B and C in China. J Gastroenterol Hepatol 2013;28 Suppl 1:7-10.

-4 Lu L, Wu T, Xiong L, Li C, Nguyen MH, Murphy DG: Analysis of HCV-6 isolates among Asian-born immigrants in North America reveals their high genetic diversity and a new subtype. Virology 2016;492:25-31. 


\section{Cellular Physiology Cell Physiol Biochem 2018;50:1832-1839 \begin{tabular}{ll|l} 
DOI: 10.1159/000494863 & $\begin{array}{l}\text { O } 2018 \text { The Author(s). Published by S. Karger AG, Basel } \\
\text { www.karger.com/cpb }\end{array}$ \\
\hline
\end{tabular} \\ Wu et al.: Unique Evolution of HCV Among the Li Ethnic in Hainan Island and a New Subtype}

5 Lu L, Li C, Fu Y, Thaikruea L, Thongswat S, Maneekarn N, Apichartpiyakul C, Hotta H, Okamoto H, Netski D, Pybus OG, Murphy D, Hagedorn CH, Nelson KE: Complete genomes for hepatitis C virus subtypes 6f, 6i, 6j and 6m: Viral genetic diversity among Thai blood donors and infected spouses. J Gen Virol 2007;88:15051518.

6 Smith DB, Bukh J, Kuiken C, Muerhoff AS, Rice CM, Stapleton JT, Simmonds P: Expanded classification of hepatitis $C$ virus into 7 genotypes and 67 subtypes: Updated criteria and genotype assignment web resource. Hepatology 2014;59:318-327.

7 Li C, Pham VH, Abe K, Lu L: Nine additional complete genome sequences of HCV genotype 6 from Vietnam including new subtypes 6xb and 6xc. Virology 2014;468-470:172-177.

-8 Cheung MC, Walker AJ, Hudson BE, Verma S, McLauchlan J, Mutimer DJ, Brown A, Gelson WT, MacDonald DC, Agarwal K, Foster GR, Irving WL: Outcomes after successful direct-acting antiviral therapy for patients with chronic hepatitis C and decompensated cirrhosis. J Hepatol 2016;65:741-747.

-9 Terrault NA, Zeuzem S, Di Bisceglie AM, Lim JK, Pockros PJ, Frazier LM, Kuo A, Lok AS, Shiffman ML, Ben AZ, Akushevich L, Vainorius M, Sulkowski MS, Fried MW, Nelson DR: Effectiveness of Ledipasvir-Sofosbuvir combination in patients with hepatitis c virus infection and factors associated with sustained virologic response. Gastroenterology 2016;151:1131-1140.

10 Rao H, Wei L, Lopez-Talavera JC, Shang J, Chen H, Li J, Xie Q, Gao Z, Wang L, Wei J, Jiang J, Sun Y, Yang R, Li H, Zhang H, Gong Z, Zhang L, Zhao L, Dou X, Niu J, You H, Chen Z, Ning Q Gong G, Wu S, Ji W, Mao Q, Tang H, Li S, Wei S, Sun J, Jiang J, Lu L, Jia J, Zhuang H: Distribution and clinical correlates of viral and host genotypes in Chinese patients with chronic hepatitis C virus infection. J Gastroenterol Hepatol 2014;29:545-553.

11 Wu T, Xiong L, Wang F, Xu X, Wang J, Lin F, Li C, Lu L, Zhou Y: A unique pattern of HCV genotype distribution on hainan island in china revealed by evolutionary analysis. Cell Physiol Biochem 2016;39:316-330.

12 Lu L, Li C, Fu Y, Gao F, Pybus OG, Abe K, Okamoto H, Hagedorn CH, Murphy D: Complete genomes of hepatitis $\mathrm{C}$ virus (HCV) subtypes 6c, 6l, 6o, 6p and 6q: Completion of a full panel of genomes for HCV genotype 6. J Gen Virol 2007;88:1519-1525.

13 Kwok S, Higuchi R: Avoiding false positives with PCR. Nature 1989;339:237-238.

14 Martin DP, Lemey P, Lott M, Moulton V, Posada D, Lefeuvre P: RDP3: A flexible and fast computer program for analyzing recombination. Bioinformatics 2010;26:2462-2463.

15 Simmonds P, Bukh J, Combet C, Deleage G, Enomoto N, Feinstone S, Halfon P, Inchauspe G, Kuiken C, Maertens G, Mizokami M, Murphy DG, Okamoto H, Pawlotsky JM, Penin F, Sablon E, Shin-I T, Stuyver LJ, Thiel HJ, Viazov S, Weiner AJ, Widell A: Consensus proposals for a unified system of nomenclature of hepatitis C virus genotypes. Hepatology 2005;42:962-973.

16 Salemi M, Vandamme AM: Hepatitis C virus evolutionary patterns studied through analysis of full-genome sequences. J Mol Evol 2002;54:62-70.

-17 Wang H, Yuan Z, Barnes E, Yuan M, Li C, Fu Y, Xia X, Li G, Newton PN, Vongsouvath M, Klenerman P, Pybus OG, Murphy D, Abe K, Lu L: Eight novel hepatitis $\mathrm{C}$ virus genomes reveal the changing taxonomic structure of genotype 6. J Gen Virol 2013;94:76-80.

18 Li C, Barnes E, Newton PN, Fu Y, Vongsouvath M, Klenerman P, Okamoto H, Abe K, Pybus OG, Lu L: An expanded taxonomy of hepatitis $C$ virus genotype 6: Characterization of 22 new full-length viral genomes. Virology 2015;476:355-363.

19 Lu L, Nakano T, He Y, Fu Y, Hagedorn CH, Robertson BH: Hepatitis C virus genotype distribution in China: Predominance of closely related subtype $1 \mathrm{~b}$ isolates and existence of new genotype 6 variants. J Med Virol 2005;75:538-549.

20 Nguyen NH, Nguyen MH: Current treatment options in patients with hepatitis c virus genotype 6. Gastroenterol Clin North Am 2015;44:871-881.

-21 Wasitthankasem R, Vongpunsawad S, Siripon N, Suya C, Chulothok P, Chaiear K, Rujirojindakul P, Kanjana S, Theamboonlers A, Tangkijvanich P, Poovorawan Y: Genotypic distribution of hepatitis C virus in Thailand and Southeast Asia. PLoS One 2015;10:e126764.

22 Mohd HK, Groeger J, Flaxman AD, Wiersma ST: Global epidemiology of hepatitis C virus infection: New estimates of age-specific antibody to HCV seroprevalence. Hepatology 2013;57:1333-1342.

23 Lee MH, Hsiao TI, Subramaniam SR, Le AK, Vu VD, Trinh HN, Zhang J, Jin M, Wong VW, Wong GL, Nguyen MH: HCV genotype 6 increased the risk for hepatocellular carcinoma among asian patients with liver cirrhosis. Am J Gastroenterol 2017;112:1111-1119. 


\section{Cellular Physiology Cell Physiol Biochem 2018;50:1832-1839 \begin{tabular}{ll|l} 
DOI: 10.1159/000494863 & O 2018 The Author(s). Published by S. Karger AG, Basel \\
www.karger.com/cpb
\end{tabular}}

Wu et al.: Unique Evolution of HCV Among the Li Ethnic in Hainan Island and a New Subtype

-24 Lim SG, Aghemo A, Chen PJ, Dan YY, Gane E, Gani R, Gish RG, Guan R, Jia JD, Lim K, Piratvisuth T, Shah S, Shiffman ML, Tacke F, Tan SS, Tanwandee T, Win KM, Yurdaydin C: Management of hepatitis C virus infection in the Asia-Pacific region: An update. Lancet Gastroenterol Hepatol 2017;2:52-62.

25 Martinello M, Amin J, Matthews GV, Dore GJ: Prevalence and disease burden of HCV coinfection in HIV cohorts in the asia pacific region: A systematic review and Meta-Analysis. AIDS Rev 2016;18:68-80.

-26 Fu Y, Qin W, Cao H, Xu R, Tan Y, Lu T, Wang H, Tong W, Rong X, Li G, Yuan M, Li C, Abe K, Lu L, Chen G: HCV 6a prevalence in Guangdong province had the origin from Vietnam and recent dissemination to other regions of China: Phylogeographic analyses. PLoS One 2012;7:e28006.

-27 Hubschen JM, Jutavijittum P, Thammavong T, Samountry B, Yousukh A, Toriyama K, Sausy A, Muller CP: High genetic diversity including potential new subtypes of hepatitis C virus genotype 6 in Lao People's Democratic Republic. Clin Microbiol Infect 2011;17:E30-E34.

28 Akkarathamrongsin S, Praianantathavorn K, Hacharoen N, Theamboonlers A, Tangkijvanich P, Poovorawan Y: Seroprevalence and genotype of hepatitis $\mathrm{C}$ virus among immigrant workers from Cambodia and Myanmar in Thailand. Intervirology 2011;54:10-16.

29 Li C, Yuan M, Lu L, Lu T, Xia W, Pham VH, Vo AX, Nguyen MH, Abe K: The genetic diversity and evolutionary history of hepatitis C virus in Vietnam. Virology 2014;468-470:197-206.

-30 An Y, Wu T, Wang M, Lu L, Li C, Zhou Y, Fu Y, Chen G: Conservation in China of a novel group of HCV variants dating to six centuries ago. Virology 2014;464-465:21-25. 